\title{
OSCILLATION OF PARTIAL FUNCTIONAL-DIFFERENTIAL EQUATIONS WITH DEVIATING ARGUMENTS
}

\author{
NORIO YOSHIDA
}

\begin{abstract}
A.bstract. A class of partial functional-differential equations with deviating arguments including parabolic equations, hyperbolic equations and beam equations is studied, and sufficient conditions are derived for all solutions of certain boundary value problem to be oscillatory in a cylindrical domain.
\end{abstract}

There has been an increasing interest in studying the oscillation of parabolic or hyperbolic equations with deviating arguments. We refer the reader to $[3,5,10,11]$ for the forced oscillation of parabolic equations with deviating arguments, and to [6, 12] for the forced oscillation of hyperbolic equations with deviating arguments. We mention in particular the paper of Onose and Yokoyama [8] which deals with higher order partial differential equations without deviating arguments. However, there appears to be no known results about oscillation of higher order partial differential equations with deviating arguments.

We are concerned with the oscillatory behavior of solutions of the higher order partial functional-differential equations with deviating arguments

$$
\begin{aligned}
& \frac{\partial^{\ell}}{\partial t^{\ell}}\left(u(x, t)+\sum_{i=1}^{M} h_{i}(t) u\left(x, \rho_{i}(t)\right)\right) \\
& -\left[\sum_{j=1}^{N} a_{j}(t) \triangle^{j} u(x, t)+\sum_{i=1}^{k} \sum_{j=1}^{N} b_{i j}(t) \triangle^{j} u\left(x, \sigma_{i j}(t)\right)\right] \\
& +c\left(x, t, u(x, t), u\left(x, \tau_{1}(t)\right), \ldots, u\left(x, \tau_{m}(t)\right), z_{1}[u](x, t), z_{2}[u](x, t)\right) \\
& =f(x, t), \quad(x, t) \in \Omega \equiv G \times(0, \infty),
\end{aligned}
$$

where $G$ is a bounded domain of $\mathbb{R}^{n}$ with piecewise smooth boundary $\partial G, \Delta$ is the Laplacian in $\mathbb{R}^{n}$ and $\Delta^{j}$ is the $j$-th iterated Laplacian. We consider the boundary condition

$$
\triangle^{j} u=\psi_{j+1} \text { on } \partial G \times(0, \infty) \quad(j=0,1, \ldots, N-1),
$$

Received November 19, 1993.

1991 Mathematics Subject Classification. 35B05, 35R10.

Key words and phrases. Oscillation, deviating arguments. 
where $\psi_{j+1} \in C\left(\partial G \times(0, \infty) ; \mathbb{R}^{1}\right) \quad(j=0,1, \ldots, N-1)$.

Our objective is to derive sufficient conditions for every solution of the boundary value problem (1), (2) to be oscillatory in $\Omega$.

It is assumed throughout this paper that:

$\left(\mathrm{H}_{1}\right) h_{i}(t) \in C^{\ell}((0, \infty) ;[0, \infty))(i=1,2, \ldots, M)$,

$a_{j}(t) \in C\left((0, \infty) ; \mathbb{R}^{1}\right)(j=1,2, \ldots, N)$,

$b_{i j}(t) \in C\left((0, \infty) ; \mathbb{R}^{1}\right)(i=1,2, \ldots, k ; j=1,2, \ldots, N)$

and $f(x, t) \in C\left(\bar{\Omega} ; \mathbb{R}^{1}\right)$;

$\left(\mathrm{H}_{2}\right) \rho_{i}(t) \in C^{\ell}\left((0, \infty) ; \mathbb{R}^{1}\right), \lim _{t \rightarrow \infty} \rho_{i}(t)=\infty(i=1,2, \ldots, M)$,

$\sigma_{i j}(t) \in C\left((0, \infty) ; \mathbb{R}^{1}\right), \lim _{t \rightarrow \infty} \sigma_{i j}(t)=\infty(i=1,2, \ldots, k ; j=1,2, \ldots, N)$,

$\tau_{i}(t) \in C\left((0, \infty) ; \mathbb{R}^{1}\right), \lim _{t \rightarrow \infty} \tau_{i}(t)=\infty(i=1,2, \ldots, m) ;$

$\left(\mathrm{H}_{3}\right) z_{1}[u](x, t)=\max _{s \in M(t)} u(x, s)$,

$z_{2}[u](x, t)=\sum_{j=1}^{m} \int_{G} K_{j}(x, t, y) h_{j}\left(u\left(y, \tau_{j}(t)\right)\right) d y$

where $M(t)$ is a closed bounded set of $[0, \infty)$ such that $\lim _{t \rightarrow \infty} \min _{s \in M(t)} s=$ $\infty, K_{j} \in C(\bar{\Omega} \times \bar{G}), K_{j} \geq 0$ in $\Omega \times G(j=1,2, \ldots, m)$, and $h_{j}(s)$ are continuous, odd functions in $\mathbb{R}^{1}$ which are nonnegative in $[0, \infty)$;

$\left(\mathrm{H}_{4}\right) c\left(x, t, \xi, \eta_{1}, \ldots, \eta_{m}, \zeta_{1}, \zeta_{2}\right) \in C\left(\bar{\Omega} \times \mathbb{R}^{m+3} ; \mathbb{R}^{1}\right)$,

$c\left(x, t, \xi, \eta_{1}, \ldots, \eta_{m}, \zeta_{1}, \zeta_{2}\right) \geq 0$ in $\Omega \times[0, \infty)^{m+3}$,

$c\left(x, t, \xi, \eta_{1}, \ldots, \eta_{m}, \zeta_{1}, \zeta_{2}\right) \leq 0$ in $\Omega \times(-\infty, 0]^{m+3}$,

where $[0, \infty)^{j}=[0, \infty) \times[0, \infty)^{j-1}$ and $(-\infty, 0]^{j}=(-\infty, 0] \times(-\infty, 0]^{j-1}$

$(j=1,2, \ldots, m+3)$.

Definition 1. By a solution of the boundary value problem (1), (2) we mean a function $u(x, t) \in C^{K}\left(\bar{G} \times\left[t_{-1}, \infty\right) ; \mathbb{R}^{1}\right) \cap C\left(\bar{G} \times\left[\tilde{t}_{-1}, \infty\right) ; \mathbb{R}^{1}\right)$ which satisfies (1), (2), where $K=\max \{\ell, 2 N\}$,

$$
\begin{aligned}
& t_{-1}=\min \left\{\min _{1 \leq i \leq M}\left\{\inf _{t \geq 0} \rho_{i}(t)\right\}, \min _{\substack{1 \leq i \leq k \\
1 \leq j \leq N}}\left\{\inf _{t \geq 0} \sigma_{i j}(t)\right\}, 0\right\}, \\
& \tilde{t}_{-1}=\min \left\{\min _{1 \leq i \leq m}\left\{\inf _{t \geq 0} \tau_{i}(t)\right\}, 0\right\}
\end{aligned}
$$

Definition 2. A solution $u$ of the boundary value problem (1), (2) is said to be oscillatory in $\Omega$ if $u$ has a zero in $G \times(t, \infty)$ for any $t>0$.

It is known that the first eigenvalue $\lambda_{1}$ of the eigenvalue problem

$$
\begin{gathered}
-\Delta w=\lambda w \quad \text { in } \quad G, \\
w=0 \quad \text { on } \quad \partial G
\end{gathered}
$$

is positive and the corresponding eigenfunction $\Phi(x)$ may be chosen so that $\Phi(x)>0$ in G. 
The following notation will be used:

$$
\begin{aligned}
& U(t)=\int_{G} u(x, t) \Phi(x) d x, \quad t \geq 0, \\
& F(t)=\int_{G} f(x, t) \Phi(x) d x, \quad t \geq 0 .
\end{aligned}
$$

Lemma 1. Let $u$ be a solution of the boundary value problem (1), (2). Then the following identity holds:

$$
\int_{G}\left(\Delta^{j} u\right) \Phi(x) d x=-\sum_{p=0}^{j-1}\left(-\lambda_{1}\right)^{p} \Psi_{j-p}(t)+\left(-\lambda_{1}\right)^{j} U(t), \quad t>0, \quad(j=1,2, \ldots, N)
$$

where

$$
\Psi_{j-p}(t)=\int_{\partial G} \psi_{j-p} \frac{\partial \Phi}{\partial \nu}(x) d S \quad(p=0,1, \ldots, j-1 ; j=1,2, \ldots, N)
$$

$\nu$ being the unit exterior normal vector to $\partial G$.

Proof. It follows from Green's formula that

$$
\begin{aligned}
\int_{G}\left(\triangle^{j} u\right) \Phi(x) d x= & \int_{\partial G}\left(\frac{\partial}{\partial \nu}\left(\triangle^{j-1} u\right) \Phi(x)-\left(\triangle^{j-1} u\right) \frac{\partial \Phi}{\partial \nu}(x)\right) d S \\
& +\int_{G}\left(\triangle^{j-1} u\right) \triangle \Phi(x) d x \\
= & -\int_{\partial G} \psi_{j} \frac{\partial \Phi}{\partial \nu}(x) d S-\lambda_{1} \int_{G}\left(\Delta^{j-1} u\right) \Phi(x) d x
\end{aligned}
$$

Analogously we obtain

$$
\int_{G}\left(\triangle^{j-1} u\right) \Phi(x) d x=-\int_{\partial G} \psi_{j-1} \frac{\partial \Phi}{\partial \nu}(x) d S-\lambda_{1} \int_{G}\left(\triangle^{j-2} u\right) \Phi(x) d x .
$$

Combining (4) with (5) yields

$$
\begin{aligned}
\int_{G}\left(\triangle^{j} u\right) \Phi(x) d x= & -\int_{\partial G} \psi_{j} \frac{\partial \Phi}{\partial \nu}(x) d S-\lambda_{1}\left(-\int_{\partial G} \psi_{j-1} \frac{\partial \Phi}{\partial \nu}(x) d S\right) \\
& +\left(-\lambda_{1}\right)^{2} \int_{G}\left(\triangle^{j-2} u\right) \Phi(x) d x .
\end{aligned}
$$

Repeating this procedure, we have

$$
\int_{G}\left(\Delta^{j} u\right) \Phi(x) d x=\sum_{p=0}^{j-1}\left(-\lambda_{1}\right)^{p}\left(-\int_{\partial G} \psi_{j-p} \frac{\partial \Phi}{\partial \nu}(x) d S\right)+\left(-\lambda_{1}\right)^{j} \int_{G} u \Phi(x) d x
$$


which is the desired identity (3).

Lemma 2. If $u>0$ in $G \times\left(t_{0}, \infty\right)$ for some $t_{0}>0$, then $U(t)$ is a positive solution of the differential inequality

$$
\frac{d^{\ell}}{d t^{\ell}}\left(y(t)+\sum_{i=1}^{M} h_{i}(t) y\left(\rho_{i}(t)\right)\right)+A(t) y(t)+\sum_{i=1}^{k} \sum_{j=1}^{N} B_{i j}(t) y\left(\sigma_{i j}(t)\right) \leq Q(t)
$$

in $[T, \infty)$ for some $T \geq t_{0}$, where

$$
\begin{aligned}
A(t)= & -\sum_{j=1}^{N} a_{j}(t)\left(-\lambda_{1}\right)^{j}, \\
B_{i j}(t)= & -b_{i j}(t)\left(-\lambda_{1}\right)^{j}, \\
Q(t)= & F(t)-\sum_{j=1}^{N} \sum_{p=0}^{j-1} a_{j}(t)\left(-\lambda_{1}\right)^{p} \Psi_{j-p}(t) \\
& -\sum_{i=1}^{k} \sum_{j=1}^{N} \sum_{p=0}^{j-1} b_{i j}(t)\left(-\lambda_{1}\right)^{p} \Psi_{j-p}\left(\sigma_{i j}(t)\right) .
\end{aligned}
$$

Proof. The hypotheses $\left(\mathrm{H}_{2}\right)$ and $\left(\mathrm{H}_{3}\right)$ imply that $u\left(x, \tau_{i}(t)\right)>0(i=1,2, \ldots, m)$, $z_{i}[u](x, t) \geq 0 \quad(i=1,2)$ in $G \times[T, \infty)$ for some $T \geq t_{0}$. Hence, it follows from the hypothesis $\left(\mathrm{H}_{4}\right)$ that

$$
\begin{aligned}
& \frac{\partial^{\ell}}{\partial t^{\ell}}\left(u(x, t)+\sum_{i=1}^{M} h_{i}(t) u\left(x, \rho_{i}(t)\right)\right) \\
& -\left[\sum_{j=1}^{N} a_{j}(t) \Delta^{j} u(x, t)+\sum_{i=1}^{k} \sum_{j=1}^{N} b_{i j}(t) \Delta^{j} u\left(x, \sigma_{i j}(t)\right)\right] \\
& \leq f(x, t), \quad(x, t) \in G \times[T, \infty) .
\end{aligned}
$$

Multiplying (7) by $\Phi(x)$ and then integrating over $G$, we observe, using Lemma 1, that

$$
\begin{aligned}
& \frac{d^{\ell}}{d t^{\ell}}\left(U(t)+\sum_{i=1}^{M} h_{i}(t) U\left(\rho_{i}(t)\right)\right) \\
& -\left[\sum_{j=1}^{N} a_{j}(t)\left(-\sum_{p=0}^{j-1}\left(-\lambda_{1}\right)^{p} \Psi_{j-p}(t)+\left(-\lambda_{1}\right)^{j} U(t)\right)\right. \\
& \left.+\sum_{i=1}^{k} \sum_{j=1}^{N} b_{i j}(t)\left(-\sum_{p=0}^{j-1}\left(-\lambda_{1}\right)^{p} \Psi_{j-p}\left(\sigma_{i j}(t)\right)+\left(-\lambda_{1}\right)^{j} U\left(\sigma_{i j}(t)\right)\right)\right] \\
& \leq F(t), \quad t \geq T,
\end{aligned}
$$


which is equivalent to

$$
\begin{aligned}
\frac{d^{\ell}}{d t^{\ell}}\left(U(t)+\sum_{i=1}^{M} h_{i}(t) U\left(\rho_{i}(t)\right)\right)-\sum_{j=1}^{N} a_{j}(t)\left(-\lambda_{1}\right)^{j} U(t) \\
\quad-\sum_{i=1}^{k} \sum_{j=1}^{N} b_{i j}(t)\left(-\lambda_{1}\right)^{j} U\left(\sigma_{i j}(t)\right) \leq Q(t), \quad t \geq T .
\end{aligned}
$$

The above inequality shows that $\mathrm{U}(\mathrm{t})$ is a positive solution of $(6)$ in $[T, \infty)$.

Theorem 1. Assume that $\left(\mathrm{H}_{1}\right)-\left(\mathrm{H}_{4}\right)$ hold. If the differential inequalities

$$
\frac{d^{\ell}}{d t^{\ell}}\left(y(t)+\sum_{i=1}^{M} h_{i}(t) y\left(\rho_{i}(t)\right)\right)+A(t) y(t)+\sum_{i=1}^{k} \sum_{j=1}^{N} B_{i j}(t) y\left(\sigma_{i j}(t)\right) \leq \pm Q(t)
$$

have no eventually positive solutions, then every solution $u$ of the problem (1), (2) is oscillatory in $\Omega$.

Proof. Suppose to the contrary that there is a solution $u$ of the problem (1), (2) which has no zero in $G \times\left(t_{0}, \infty\right)$ for some $t_{0}>0$. First we suppose that $u>0$ in $G \times\left(t_{0}, \infty\right)$. Lemma 2 implies that $U(t)$ is an eventually positive solution of (6). This contradicts the hypothesis. If $u<0$ in $G \times\left(t_{0}, \infty\right)$, then $-U(t)$ is a positive solution of the differential inequality

$$
\frac{d^{\ell}}{d t^{\ell}}\left(y(t)+\sum_{i=1}^{M} h_{i}(t) y\left(\rho_{i}(t)\right)\right)+A(t) y(t)+\sum_{i=1}^{k} \sum_{j=1}^{N} B_{i j}(t) y\left(\sigma_{i j}(t)\right) \leq-Q(t), \quad t \geq T
$$

for some $T \geq t_{0}$. This contradicts the hypothesis and the proof is complete.

Lemma 3. The differential inequality (6) has no eventually positive solution if for some $t_{0}>0$

$$
\begin{aligned}
& A(t) \geq 0 \quad \text { in }\left[t_{0}, \infty\right), \\
& B_{i j}(t) \geq 0 \quad \text { in }\left[t_{0}, \infty\right)(i=1,2, \ldots, k ; j=1,2, \ldots, N), \\
& \liminf _{t \rightarrow \infty} \int_{T}^{t}\left(1-\frac{s}{t}\right)^{\ell-1} Q(s) d s=-\infty \quad \text { for all large } T .
\end{aligned}
$$

Proof. Suppose that there is a positive solution $y(t)$ of $(6)$ in $\left[T_{0}, \infty\right)$ for some $T_{0}>0$. From the hypothesis we see that

$$
\frac{d^{\ell}}{d t^{\ell}}\left(y(t)+\sum_{i=1}^{M} h_{i}(t) y\left(\rho_{i}(t)\right)\right) \leq Q(t), \quad t \geq T
$$


for some $T \geq \max \left\{t_{0}, T_{0}\right\}$. Integrating (9) over $[T, t]$ yields

$$
Y^{(\ell-1)}(t)-Y^{(\ell-1)}(T) \leq \int_{T}^{t} Q(s) d s, \quad t \geq T
$$

where $Y(t) \equiv y(t)+\sum_{i=1}^{M} h_{i}(t) y\left(\rho_{i}(t)\right)$. We integrate (10) over $[T, t]$ to obtain

$$
Y^{(\ell-2)}(t)-Y^{(\ell-2)}(T)-Y^{(\ell-1)}(T)(t-T) \leq \int_{T}^{t}\left(\int_{T}^{s_{2}} Q\left(s_{1}\right) d s_{1}\right) d s_{2}, \quad t \geq T .
$$

Repetition of this procedure yields finally the inequality

$$
Y(t)-\sum_{i=0}^{\ell-1} \frac{(t-T)^{i}}{i !} Y^{(i)}(T) \leq \int_{T}^{t} \int_{T}^{s_{\ell}} \cdots \int_{T}^{s_{2}} Q\left(s_{1}\right) d s_{1} \cdots d s_{\ell-1} d s_{\ell}, \quad t \geq T .
$$

It is easily seen that

$$
\int_{T}^{t} \int_{T}^{s_{\ell}} \cdots \int_{T}^{s_{2}} Q\left(s_{1}\right) d s_{1} \cdots d s_{\ell-1} d s_{\ell}=\frac{1}{(\ell-1) !} \int_{T}^{t}(t-s)^{\ell-1} Q(s) d s .
$$

Substituting (12) into (11) and then dividing (11) by $t^{\ell-1}$, we obtain

$$
\frac{Y(t)}{t^{\ell-1}}-\sum_{i=0}^{\ell-1} \frac{Y^{(i)}(T)}{i !}\left(1-\frac{T}{t}\right)^{i} \frac{1}{t^{\ell-i-1}} \leq \frac{1}{(\ell-1) !} \int_{T}^{t}\left(1-\frac{s}{t}\right)^{\ell-1} Q(s) d s, \quad t \geq T .
$$

Since $Y(t)>0$ in $\left[T_{1}, \infty\right)$ for some $T_{1} \geq T$, the left hand side of (13) is bounded from below in $\left[T_{1}, \infty\right)$. However, the hypothesis implies that the left hand side of $(13)$ is not bounded from below. The contradiction establishes the theorem.

Next we consider the case where $Q(t) \equiv 0, h_{i}(t) \equiv 0 \quad(i=1,2, \ldots, M)$, i. e.

$$
\frac{d^{\ell}}{d t^{\ell}} y(t)+A(t) y(t)+\sum_{i=1}^{k} \sum_{j=1}^{N} B_{i j}(t) y\left(\sigma_{i j}(t)\right) \leq 0
$$

Lemma 4. Let $\ell$ be a positive even integer. The differential inequality (14) has no eventually positive solution if for some $t_{0}>0$

$$
\begin{aligned}
& A(t)>0 \quad \text { in }\left[t_{0}, \infty\right), \\
& B_{i j}(t) \geq 0 \quad \text { in }\left[t_{0}, \infty\right) \quad(i=1,2, \ldots, k ; j=1,2, \ldots, N), \\
& \int^{\infty} t^{\ell-1-\varepsilon} A(t) d t=\infty \quad \text { for some } \varepsilon>0 .
\end{aligned}
$$

Proof. By the hypothesis the differential equation

$$
\frac{d^{\ell}}{d t^{\ell}} y(t)+A(t) y(t)=0
$$


has no eventually positive solution (see Mikusiński [4]). Using the results of Kartsatos [2] and Onose [7], we observe that the differential inequality

$$
\frac{d^{\ell}}{d t^{\ell}} y(t)+A(t) y(t) \leq 0
$$

has no eventually positive solution. Since $B_{i j}(t) \geq 0 \quad(i=1,2, \ldots, k ; j=1,2, \ldots, N)$ in $\left[t_{0}, \infty\right),(14)$ has no eventually positive solution.

Theorem 2. Assume that $\left(\mathrm{H}_{1}\right)-\left(\mathrm{H}_{4}\right)$ hold. Every solution $u$ of the problem $(1),(2)$ is oscillatory in $\Omega$ if for some $t_{0}>0$

$$
\begin{aligned}
& A(t) \geq 0 \quad \text { in }\left[t_{0}, \infty\right), \\
& B_{i j}(t) \geq 0 \quad \text { in }\left[t_{0}, \infty\right) \quad(i=1,2, \ldots, k ; j=1,2, \ldots, N), \\
& \liminf _{t \rightarrow \infty} \int_{T}^{t}\left(1-\frac{s}{t}\right)^{\ell-1} Q(s) d s=-\infty \quad \text { for all large } T, \\
& \limsup _{t \rightarrow \infty} \int_{T}^{t}\left(1-\frac{s}{t}\right)^{\ell-1} Q(s) d s=\infty \quad \text { for all large } T .
\end{aligned}
$$

Proof. Since

$$
\liminf _{t \rightarrow \infty} \int_{T}^{t}\left(1-\frac{s}{t}\right)^{\ell-1}( \pm Q(s)) d s=-\infty
$$

the conclusion follows from Theorem 1 and Lemma 3.

Theorem 3. Assume that $\left(\mathrm{H}_{1}\right)-\left(\mathrm{H}_{4}\right)$ hold. Let $Q(t) \equiv 0, h_{i}(t) \equiv 0 \quad(i=$ $1,2, \ldots, M)$, and let $\ell$ be a positive even integer. Every solution $u$ of the problem $(1),(2)$ is oscillatory in $\Omega$ if for some $t_{0}>0$

$$
\begin{aligned}
& A(t)>0 \quad \text { in }\left[t_{0}, \infty\right), \\
& B_{i j}(t) \geq 0 \quad \text { in }\left[t_{0}, \infty\right) \quad(i=1,2, \ldots, k ; j=1,2, \ldots, N), \\
& \int^{\infty} t^{\ell-1-\varepsilon} A(t) d t=\infty \quad \text { for some } \varepsilon>0 .
\end{aligned}
$$

Proof. Since (8) reduces to (14), the conclusion follows from Theorem 1 and Lemma 4.

Remark 1. If $\ell=N=2, n=1, a_{2}(t)=-\alpha$ ( $\alpha$ is a positive constant), then the equation (1) reduces to the beam equation with deviating arguments

$$
\begin{aligned}
& \frac{\partial^{2}}{\partial t^{2}}\left(u(x, t)+\sum_{i=1}^{M} h_{i}(t) u\left(x, \rho_{i}(t)\right)\right)+\alpha u_{x x x x}(x, t)-\sum_{i=1}^{k} b_{i 2}(t) u_{x x x x}\left(x, \sigma_{i 2}(t)\right) \\
& -a_{1}(t) u_{x x}(x, t)-\sum_{i=1}^{k} b_{i 1}(t) u_{x x}\left(x, \sigma_{i 1}(t)\right) \\
& +c\left(x, t, u(x, t), u\left(x, \tau_{1}(t)\right), \ldots, u\left(x, \tau_{m}(t)\right), z_{1}[u](x, t), z_{2}[u](x, t)\right)=f(x, t)
\end{aligned}
$$


for $(x, t) \in(0, L) \times(0, \infty)$, where $L$ is a positive number. In case $\psi_{1}=\psi_{2}=0$ on $\partial G \times(0, \infty)$, the boundary condition (2) reduces to the hinged ends

$$
u(0, t)=u_{x x}(0, t)=u(L, t)=u_{x x}(L, t)=0, \quad t>0 .
$$

The above beam equation without deviating arguments was studied by Feireisl and Herrmann [1] and the author [9].

Remark 2. We cannot apply Theorem 2 to the case where $f(x, t) \equiv 0$ in $\Omega$ and $\psi_{j} \equiv 0$ on $\partial G \times(0, \infty) \quad(j=1,2, \ldots, N)$. However, Theorem 3 can be applied to this case.

Remark 3. In the case where $h_{i}(t) \equiv 0$ in $(0, \infty) \quad(i=1,2, \ldots, N), f(x, t) \equiv 0$ in $\Omega, \psi_{1} \equiv 0$ on $\partial G \times(0, \infty)$ and $N=1$, the equation (1) is closely related to the higher order partial differential equation which was investigated by Onose and Yokoyama [8].

Remark 4. When specialized to the case where $\ell=M=N=k=1$, Theorems 1 and 2 reduce to Theorems 2 and 5 of Mishev [5], respectively. When $\ell=2, M=N=$ $k=1$, Theorems 1 and 2 reduce to Theorems 1 and 4 of Mishev [6], respectively.

Remark 5. If $\ell=N=1$, Theorem 2 is closely connected with a result of the author [11, Theorem 1].

Example 1. Let us consider the problem

$$
\begin{aligned}
& \frac{\partial^{3}}{\partial t^{3}}\left(u(x, t)+u\left(x, t-\frac{\pi}{2}\right)\right)-\left[u_{x x}(x, t)+u_{x x}\left(x, t-\frac{3}{2} \pi\right)\right]+t^{3} u\left(x, t-\frac{\pi}{2}\right) \\
&=(\sin x) t^{3} \sin t, \quad(x, t) \in(0, \pi) \times(0, \infty), \\
& u(0, t)=u(\pi, t)=0, \quad t>0 .
\end{aligned}
$$

Here $n=1, G=(0, \pi), \ell=3, M=N=k=m=1, h_{1}(t)=a_{1}(t)=b_{11}(t)=1$, $\rho_{1}(t)=t-\frac{\pi}{2}, \sigma_{11}(t)=t-\frac{3}{2} \pi, \tau_{1}(t)=t-\frac{\pi}{2}, f(x, t)=(\sin x) t^{3} \sin t$ and $\psi_{1}=0$. It is easily seen that $\lambda_{1}=1, \Phi(x)=\sin x, \Psi_{1}(t)=0$ and $Q(t)=F(t)=\frac{\pi}{2} t^{3} \sin t$. A simple calculation shows that

$$
\int_{T}^{t}\left(1-\frac{s}{t}\right)^{2} Q(s) d s=\pi t \cos t+B(t, T),
$$

where $B(t, T)$ is bounded as $t$ tends to infinity. Since $A(t)=\lambda_{1} a_{1}(t)=1>0, B_{11}(t)=$ $\lambda_{1} b_{11}(t)=1>0$ and

$$
\begin{gathered}
\liminf _{t \rightarrow \infty} \int_{T}^{t}\left(1-\frac{s}{t}\right)^{2} Q(s) d s=-\infty \\
\limsup _{t \rightarrow \infty} \int_{T}^{t}\left(1-\frac{s}{t}\right)^{2} Q(s) d s=\infty
\end{gathered}
$$


for all large $T$, it follows from Theorem 2 that every solution $u$ of the problem (15), (16) is oscillatory in $(0, \pi) \times(0, \infty)$. One such solution is $u=\sin x \cdot \cos t$.

Example 2. We consider the problem

$$
\begin{gathered}
\frac{\partial^{4}}{\partial t^{4}} u(x, t)-\left[-u_{x x x x}(x, t)-u_{x x x x}(x, t-\pi)\right]+u(x, t-\pi)=0,(x, t) \in(0, \pi) \times(0, \infty), \\
u(0, t)=u_{x x}(0, t)=u(\pi, t)=u_{x x}(\pi, t)=0, \quad t>0 .
\end{gathered}
$$

Here $n=1, G=(0, \pi), \ell=4, N=2, k=m=1, a_{1}(t)=0, a_{2}(t)=-1, b_{11}(t)=0$, $b_{12}(t)=-1, \sigma_{12}(t)=\tau_{1}(t)=t-\pi, f(x, t)=0, \psi_{1}=\psi_{2}=0$ and $\lambda_{1}=1$. Since $A(t)=-\lambda_{1}^{2} a_{2}(t)=1>0, B_{11}(t)=0, B_{12}(t)=-\lambda_{1}^{2} b_{12}(t)=1>0, Q(t)=0$ and

$$
\int^{\infty} t^{4-1-\varepsilon} A(t) d t=\infty
$$

for any $\varepsilon$ with $0<\varepsilon \leq 4$, Theorem 3 implies that every solution $u$ of the problem (17), (18) is oscillatory in $(0, \pi) \times(0, \infty)$. Indeed, $u=\sin x \cdot \sin t$ is such a solution.

\section{References}

[1] E. Feireisl and L. Herrmann, "Oscillations of a nonlinearly damped extensible beam," Appl. Math., 37 (1992), 469-478.

[2] A. G. Kartsatos, "On $n$th order differential inequalities," J. Math. Anal. Appl., 52 (1975), 1-9.

[3] Kusano T. and N. Yoshida, "Oscillation of parabolic equations with oscillating coefficients," $H i$ roshima Math. J., 24 (1994), 123-133.

[4] J. Mikusiński, "On Fite's oscillation theorems," Colloq. Math., 2 (1951), 34-39.

[5] D. P. Mishev, "Oscillation of the solutions of parabolic differential equations of neutral type with "maxima" " Godishnik VUZ Appl. Math., 25 (1989), 19-28.

[6] D. P. Mishev, "Oscillation of the solutions of hyperbolic differential equations of neutral type with "maxima"," Godishnik VUZ Appl. Math., 25 (1989), 9-18.

[7] H. Onose, "A comparison theorem and the forced oscillation," Bull. Austral. Math. Soc., 13 (1975), 13-19.

[8] H. Onose and E. Yokoyama, "Oscillation of partial differential inequalities," Tamkang J. Math., 7 (1976), 67-70.

[9] N. Yoshida, "Forced oscillations of extensible beams," SIAM J. Math. Anal., 16 (1985), 211-220.

[10] N. Yoshida, "Forced oscillations of solutions of parabolic equations," Bull. Austral. Math. Soc., 36 (1987), 289-294.

[11] N. Yoshida, "Forced oscillations of parabolic equations with deviating arguments," Math. J. Toyama Univ., 15 (1992), 131-142.

[12] N. Yoshida, " On the zeros of solutions of hyperbolic equations with deviating arguments," Math. J. Toyama Univ., 16 (1993), 125-133.

Department of Mathematics, Faculty of Science, Toyama University, Toyama 930, Japan. 